\title{
Acute-on-chronic liver failure: a new syndrome in cirrhosis
}

\author{
Richard Moreau ${ }^{1,2,3,4,}$ \\ 'Inserm, U1149, Centre de Recherche sur l'Inflammation (CRI), Clichy and Paris; ${ }^{2}$ UMRS1149, Université Paris Diderot-Paris 7, Paris; \\ ${ }^{3}$ Département Hospitalo-Universitaire (DHU) UNITY, Service d'Hépatologie, Hôpital Beaujon, Assistance Publique-Hôpitaux de Paris, \\ Clichy; ${ }^{4}$ Laboratoire d’Excellence Inflamex, ComUE Sorbonne Paris Cité, Paris, France
}

Patients with cirrhosis who are hospitalized for an acute decompensation (AD) and also have organ failure(s) are at high risk of short-term death. These patients have a syndrome called Acute-on-Chronic Liver Failure (ACLF). ACLF is now considered as a new syndrome that it is distinct from "mere" AD not only because of the presence of organ failure(s) and high short-term mortality but also because of younger age, higher prevalence of alcoholic etiology of cirrhosis, higher prevalence of some precipitants (such as bacterial infections, active alcoholism), and more intense systemic inflammatory response. ACLF is a new syndrome also because severe sepsis or severe alcoholic hepatitis do not account for $100 \%$ of the observed cases; in fact, almost 50\% of the cases are of "unknown" origin. In other words, severe sepsis, severe alcoholic hepatitis and ACLF of "unknown origin" are subcategories of the syndrome. (Clin Mol Hepatol 2016;22:1-6)

Keywords: Cirrhosis; Inflammation; Organ failures

\section{INTRODUCTION}

Patients with cirrhosis can rapidly develop an acute decompensation (AD), i.e., new onset ascites, hepatic encephalopathy, gastrointestinal hemorrhage, bacterial infection, or any combination of these. The development of AD usually leads patients to the hospital. Patients who are admitted for AD can be divided into two groups: the first one includes patients who have organ failure(s) while the second group is composed of patients without organ failures. ${ }^{2}$ Patients with cirrhosis and organ failure(s) have high in-hospital mortality ${ }^{3-5}$ and has long been considered as having a syndrome called acute-on-chronic liver failure (ACLF). ${ }^{2}$ It is important to note that major studies aiming to describe the natural history of cirrhosis and establish markers of prognosis considered only the compensated and decompensated states of the disease but did not consider the development of organ failures in their analyses. ${ }^{6}$ This lacuna is explained by the fact that at that time investigators did not have any definition of ACLF, based on evidence or expert opinion. Since these studies, different definitions of ACLF based on expert opinions have been suggested. ${ }^{7.9}$ In 2013, the European Association for the Study of the Liver (EASL)chronic liver failure (CLIF) Consortium published the first results of the EASL-CLIF Acute-oN-ChrONic Liver Failure In Cirrhosis (CANONIC) study which was a multicenter, European, prospective

\footnotetext{
Abbreviations:

ACLF, acute-on-chronic liver failure; AD, acute decompensation; CLIF, chronic liver failure; CANONIC, EASL-CLIF Acute-oN-ChrONic liver failure in cirrhosis; SOFA, Sequential Organ Failure Assessment; MELD, Model for End-Stage Liver Disease; INR, International Normalized Ratio; $\mathrm{PaO}_{2}$, partial pressure of arterial oxygen; $\mathrm{FIO}_{2}$, fraction of inspired oxygen; $\mathrm{SpO}_{2}$, pulse oximetry saturation; $\mathrm{HCV}$, hepatitis $\mathrm{C}$ virus; $\mathrm{HBV}$, hepatitis B virus; WCC, white-cell count; CRP, C-reactive protein; PRR, pattern-recognition receptor; PAMP, pathogen-associated molecular patterns; NOD, nucleotide-binding oligomerization domain; TLR, Tolllike receptor; DAMP, danger-associated molecular pattern
}

\section{Corresponding author : Richard Moreau}

Inserm, U1149, Centre de Recherche sur I'Inflammation (CRI), Faculté de Médecine Bichat, 16 rue Henri Huchard, 75018 Paris, France Tel: +33-157277510, Fax: +33-157277471 E-mail: Richard.moreau@inserm.fr 
observational study of patients with cirrhosis admitted for an AD.' The results of this study allowed to establish an evidence-based definition of ACLF and doing this clearly showed that ACLF is distinct from mere $A D$, and is a new syndrome which is the final common pathway driving patients with cirrhosis to death. ${ }^{1,10,11}$ The aim of this review article is to comment on the novelty of the ACLF syndrome described by the EASL-CLIF Consortium.

\section{DEFINING ORGAN FAILURE IN CIRRHOSIS}

The Sequential Organ Failure Assessment (SOFA) scale which is used to diagnose organ dysfunctions and failures in general intensive care units (ICUS) ${ }^{12}$ has also been used in patients with cirrhosis admitted to the ICU. ${ }^{4,5}$ The SOFA score was found to be a better predictor of short-term prognosis of patients with cirrhosis than were liver-specific scores (the Child-Pugh and MELD scores). ${ }^{4,5}$ The investigators of the CANONIC study used a modified SOFA scale, called CLIF-SOFA, which was pre-specified in the study protocol. ${ }^{1}$ Like the original scale, ${ }^{12}$ the CLIF-SOFA scale assessed the function of six organ-systems (liver, kidneys, brain, coagulation, circulation, and respiration) but also included some markers specific for cirrhosis (Table 1). ${ }^{1}$ Each organ-system received a sub-score ranging from zero (normal) to four (most abnormal). A total CLIF-SOFA score ranging from zero to 24 was calculated. The definitions for organ failures based on the CLIF-SOFA scale were as follows: 1) Liver failure was defined by serum biliru- bin levels of $12.0 \mathrm{mg} / \mathrm{dL}$ or more; 2) Kidney failure was defined by serum creatinine levels of $2.0 \mathrm{mg} / \mathrm{dL}$ or more, or the use of renalreplacement therapy; 3) Cerebral failure was defined by grade III or IV hepatic encephalopathy according to the West Haven classification; 4) Coagulation failure was defined by an International Normalized Ratio (INR) of more than 2.5 and/or platelet count of $20 \times 10^{9} / \mathrm{L}$ or less; 5) Circulatory failure was defined by the use of vasopressin (including terlipressin) to maintain arterial pressure; 6) Respiratory failure was defined by a ratio of partial pressure of arterial oxygen to fraction of inspired oxygen $\left(\mathrm{FiO}_{2}\right)$ of 200 or less or a pulse oximetry saturation $\left(\mathrm{SpO}_{2}\right)$ to $\mathrm{FiO}_{2}$ ratio of 200 or less (Table 1).

Recently the CLIF-SOFA scale has been simplified giving rise to the CLIF Consortium Organ Failure (OF) score (CLIF-C OFs) in which each of the six organ/system receive a score ranging from 1 to $3 .^{13}$ The CLIF-C OFs was found to be more effective than the Model for end-stage liver disease (MELD) score in predicting mortality at 28 and 90 days. $^{13}$

\section{ORGAN FAILURES AND POOR SHORT-TERM OUTCOME DIFFERENTIATE PATIENTS WITH ACLF FROM THOSE WITH “MERE” AD}

The CANONIC study reveals that ACLF is a new clinical entity, distinct from "mere" AD. Patients with cirrhosis admitted to the hos-

Table 1. The Chronic Liver Failure (CLIF)-Sequential Organ Failure Assessment (SOFA) Scale*

\begin{tabular}{|c|c|c|c|c|c|}
\hline \multirow{2}{*}{ Organ/system } & \multicolumn{5}{|c|}{ Score } \\
\hline & 0 & 1 & 2 & 3 & 4 \\
\hline Liver; Bilirubin, mg/dL & $<1.2$ & $\geq 1.2-<2.0$ & $\geq 2.0-<6.0$ & $\geq 6.0-<12.0$ & $\geq 12.0$ \\
\hline \multirow[t]{2}{*}{ Kidney; Creatinine, mg/dL } & $<1.2$ & $\geq 1.2-<2.0$ & $\geq 2.0-<3.5$ & $\geq 3.5-<5.0$ & $\geq 5.0$ \\
\hline & & & \multicolumn{3}{|c|}{ or use of renal-replacement therapy } \\
\hline Cerebral; HE grade $^{\dagger}$ & No HE & 1 & $\|$ & III & IV \\
\hline Coagulation; INR ${ }^{\ddagger}$ & $<1.1$ & $\geq 1.1-<1.25$ & $\geq 1.25-<1.5$ & $\geq 1.5-<2.5$ & $\geq 2.5$ or Platelets $\geq 20 \times 10^{9} / \mathrm{L}$ \\
\hline Circulation; MAP mmHg & $\geq 70$ & $<70$ & $\begin{array}{l}\text { Dopamine } \leq 5 \text { or } \\
\text { Dobutamine or } \\
\text { Terlipressin }^{\S}\end{array}$ & $\begin{array}{l}\text { Dopamine }>5 \text { or } \\
E \leq 0.1 \text { or } \\
N E \leq 0.1\end{array}$ & $\begin{array}{c}\text { Dopamine }>15 \text { or } \\
E>0.1 \text { or } \\
N E>0.1\end{array}$ \\
\hline $\begin{array}{l}\text { Lungs; } \mathrm{PaO}_{2} / \mathrm{FiO}_{2} \text { : or } \\
\mathrm{SpO}_{2} / \mathrm{FiO}_{2}{ }^{\pi}\end{array}$ & $\begin{array}{l}>400 \\
>512\end{array}$ & $\begin{array}{l}>300-\leq 400 \\
>357-\leq 512\end{array}$ & $\begin{array}{l}>200-\leq 300 \\
>214-\leq 357\end{array}$ & $\begin{array}{l}>100-\leq 200 \\
>89-\leq 214\end{array}$ & $\begin{array}{l}\leq 100 \\
\leq 89\end{array}$ \\
\hline
\end{tabular}

HE denotes hepatic encephalopathy; INR, International Normalized Ratio; MAP, mean arterial pressure; $E_{1}$ epinephrine; $\mathrm{NE}_{1}$ norepinephrine; $\mathrm{PaO}_{2}$, partial pressure of arterial oxygen; $\mathrm{FIO}_{2}$, fraction of inspired oxygen; $\mathrm{SpO}_{2}$, pulse oximetry saturation.

${ }^{*}$ Adapted from ref. 1. The highlighted area in violet depicts the diagnostic criteria for organ failures.

†The CLIF-SOFA scale used West Have classification while the original SOFA scale used the Coma Glasgow score.

${ }^{\ddagger}$ INR was not included in the original SOFA scale.

${ }^{5}$ Terlipressin use was not taken into account in the original SOFA scale; doses for $\mathrm{E}$ and NE are expressed in $\mu \mathrm{g} / \mathrm{kg} . \mathrm{min}$.

"The $\mathrm{SpO}_{2} / \mathrm{FiO}_{2}$ ratio was not included in the original SOFA scale. 
pital for AD can be categorized into four different groups: no ACLF (i.e., "mere" AD), ACLF grade 1, grade 2 and grade 3 (Table 2). The diagnosis of absence or presence of ACLF is simple when the number of organ failures is 0,2 , or 3 or more. Patients with no organ failure (no ACLF) have a very low 28-day mortality ( 5\%); in contrast, patients with two organ failures (ACLF grade 2) or patients with three organ failures or more (ACLF grade 3 ) have a high mortality of $32 \%$ and $\sim 80 \%$, respectively (Table 2 ).

Patients with a single organ failure are not necessarily considered as having ACLF grade 1 (Table 2). In fact, only patients with single kidney failure, who have a 28 -day mortality of $~ 20 \%$, have ACLF grade 1. For patients with a single "non-kidney" organ failure, the absence and the presence of ACLF relies on the presence or absence of kidney dysfunction (i.e., serum creatinine levels ranging from $1.5 \mathrm{mg} / \mathrm{dL}$ to $1.9 \mathrm{mg} / \mathrm{dL}$ ) and/or mild-to-moderate hepatic encephalopathy (i.e., encephalopathy grade 1-2). Patients with a single "non-kidney" organ failure who have serum creatinine levels $<1.5 \mathrm{mg} / \mathrm{dL}$, do not have ACLF group, their mortality being $5-8 \%$ (Table 2). Of note, patients with single organ failure (liver, coagulation, circulation or respiration) who also have kidney dysfunction or mild-to-moderate hepatic encephalopathy or both as well as those with single cerebral failure who also have kidney dysfunction have ACLF grade 1 because their 28-day mortality is high (20-30\%).

The evolution of patients with ACLF also shows that this syndrome is a distinct clinical entity that closely correlates with patients' severity. First, among patients who are admitted for an acute decompensation and subsequently die, ACLF grade 3 is present in all patients prior to death. ${ }^{10}$ Second, among patients who have ACLF grade 1 on admission, 50\% improve (i.e., recover to the "no-ACLF" status) and survive and $30 \%$ progress to ACLF grade 3 and die. ${ }^{10}$ Third, most patients with ACLF grade 3 at entry develop new organ failures during follow-up and die. However, it is interesting to note that $16 \%$ of patients with ACLF grade 3 on admission improve and recover to a "no ACLF" status. ${ }^{10}$ Fourth, that 28-day mortality is not nil $(\sim 5 \%)$ in patients without ACLF on admission is explained by the fact that some of these patients have developed "in-hospital ACLF" progressing to ACLF grade 3 and death. ${ }^{1,10}$ In contrast, patients without ACLF on admission and who remain "ACLF-free" during the following 28 days have a very low short-term mortality $(<2 \%)$.

Table 2. Definition of the presence or absence of ACLF and of ACLF Grades*

\section{Definitions}

Absence of ACLF 28-day transplant-free mortality rate (\%)

Defined by one the following 3 options:

1) No organ failure

2) Single organ failure (liver, coagulation, circulation, lungs) in patients with serum creatinine levels $<1.5 \mathrm{mg} / \mathrm{dL}$ and no hepatic encephalopathy ${ }^{\ddagger}$

3) Single cerebral failure in patients with serum creatinine levels $<1.5 \mathrm{mg} / \mathrm{dL}$

ACLF grade 1

22.1

Defined by one the following 3 options:

1) Single kidney failure

2) Single organ failure (liver, coagulation, circulation, lungs) in patients with serum creatinine levels ranging from $1.5 \mathrm{mg} / \mathrm{dL}$ to $1.9 \mathrm{mg} / \mathrm{dL}$ and/or grade $1-2$ hepatic encephalopathy ${ }^{\ddagger}$

3) Single cerebral failure in patients with serum creatinine levels ranging from $1.5 \mathrm{mg} / \mathrm{dL}$ to $1.9 \mathrm{mg} / \mathrm{dL}$

ACLF grade 2

Defined by the presence of two organ failures

ACLF grade 3

78.6

Defined by the presence of three organ failures or more

ACLF, acute-on-chronic liver failure.

*Adapted from ref. 1. For definition of absence or presence of organ failures, please see Table 1.

${ }^{\dagger}$ Twenty-eight-day transplant-free mortality rate is $1.9 \%$ in patients who did not have ACLF at enrollment and did not develop ACLF during the 28-day postenrollment period.

${ }^{\ddagger}$ According to West Haven classification. 


\section{CONTEXTUAL FEATURES THAT DISTINGUISH PATIENTS WITH ACLF}

Patients with ACLF are younger. In Europe, ACLF is observed mainly in patients with alcoholic cirrhosis; interestingly hepatitis $C$ virus (HCV)-related cirrhosis is lower in patients with ACLF than in those without.' In Asia, hepatitis B virus (HBV) is a common cause of $A D{ }^{7}$. Among these patients, sub-massive hepatic necrosis was found to be a hallmark in patients with HBV-associated ACLF that distinguish them from those with "mere" AD. ${ }^{14}$

ACLF is distinct from "mere" decompensation in terms of precipitating events of AD. For example, bacterial infection, in particular bacterial sepsis, is more common in patients with ACLF than in those without. ${ }^{1,15}$ Active alcoholism during the last three months is another precipitating event associated with ACLF. In other words, severe alcoholic hepatitis is a form of ACLF. Finally, there are patients in whom ACLF is triggered by the insertion of a TIPS, acute toxic or viral hepatitis superimposed to cirrhosis, major surgery or large volume paracentesis without intravenous albumin administration, but the frequency of this is low.

It might be of importance to distinguish hepatic-ACLF (e.g., that triggered by HBV reactivation or alcoholic hepatitis) from extrahepatic-ACLF (e.g., that associated with bacterial sepsis). Indeed, it has recently been shown that patients with extrahepatic-ACLF have significantly higher 90-day and 1-year mortality than patients with hepatic ACLF (90-day: $59 \%$ vs. $68 \%$; 1 -year: $64 \%$ vs. $75 \%){ }^{16}$

\section{SYSTEMIC INFLAMMATION IS A HALLMARK OF ACLF}

Systemic inflammation is closely associated with ACLF. Indeed, among patients with acutely decompensated cirrhosis, white-cell count (WCC) as well as plasma levels of C-reactive protein (CRP)' and major cytokines (i.e., interleukin (IL)-6, tumor necrosis factor (TNF)- $\alpha$ ) or chemokines (e.g., IL-8) are higher in patients with ACLF than in those without. ${ }^{1,17}$ Moreover, an increase in WCC and CRP levels has been shown across ACLF grades.' An excessive inflammatory response of the host can cause tissue damage (a process called immunopathology) and organ dysfunction or failure. ${ }^{18}$ Together these findings suggest that excessive inflammation may play a crucial role in the development of ACLF. ${ }^{19,20}$

The underlying mechanisms explaining a more intense systemic inflammatory response in patients with ACLF relative to patients without are unclear. ${ }^{20}$ Younger age of the former may be an im- portant factor because the immune response is known to be more marked in young people. ${ }^{18}$ Genetic factors may also play a role. Patients with cirrhosis who exhibit single-nucleotide polymorphism in genes encoding innate pattern-recognition receptors (PRRS, such as, NOD2, TLR4, TLR2) or a nuclear receptor (NR1H4 encoding FXR) have an increased risk of severe bacterial infection, in particular spontaneous bacterial peritonitis. ${ }^{21}$ Of note, proteins encoded by NOD2, TLR4, and TLR2 are in charge of detecting unique bacterial molecular structures called pathogen-associated molecular patterns (PAMPs). ${ }^{19,20}$ PAMPs detection by PRRs induce inflammation. ${ }^{19,20}$ The impact of these gene variants on the induction of inflammation by PAMPs should be studies in patients with cirrhosis. Patients with severe alcoholic hepatitis have higher expression of intrahepatic CXCL chemokines (e.g., IL-8) relative to patients with less pronounced alcoholic hepatitis. ${ }^{22} \mathrm{CXCL}$ chemokines are pro-inflammatory, neutrophil-attracting cues. Neutrophils can cause liver pathology. ${ }^{23}$ However, the mechanisms explaining higher CXCL chemokines in livers with severe alcoholic hepatitis are poorly understood. ${ }^{23}$ In these livers, the level of oxidative stress is high, in particular in hepatocytes. ${ }^{23} \mathrm{~A}$ translocation of intestinal bacterial components (i.e., PAMPs) may occur in alcoholic hepatitis. ${ }^{23}$ PAMPs can reach the liver via the portal vein. PAMPs are unique molecular structures that are detected by dedicated PRRs expressed in innate immune cells. ${ }^{24}$ Both, oxidative stress and bacterial PAMPs, can stimulate resident macrophages (Kupffer cells) to produce CXCL chemokines among others. ${ }^{24} \mathrm{Fu}$ ture studies are needed to investigate the respective role of hepatocyte oxidative stress and of bacterial PAMPs in the development of severe alcoholic hepatitis.

An intriguing finding is that patients with ACLF and no identifiable trigger for this syndrome also develop an excessive inflammatory response compared to corresponding patients without ACLF.' In this case systemic inflammation might be a result of intestinal translocation, not of viable bacteria (that would cause infection), but bacterial PAMPs. ${ }^{20}$ In cirrhosis, evidence has been found for a translocation of PAMPs such lipopolysaccharide (LPS) or bacterial DNA. ${ }^{19-21}$ LPS is recognized by a PRR called Toll-like receptor (TLR) 4 and bacterial DNA by TLR9. ${ }^{24}$ As mentioned earlier, PAMP detection by PRRs results in the induction of inflammatory molecules. ${ }^{24}$ Therefore, bacterial PAMP translocation may induce inflammation in the absence of any overt infection. ACLF of "unknown origin" may also be caused by non-microbial (i.e., endogenous) molecules that are released by necrotic cells. Once released in the extracellular milieu, these molecules called dangerassociated molecular patterns (DAMPs) can be recognized by 
some PRRs and trigger inflammation. ${ }^{19,20,25}$ An example of DAMP released by necrotic cells is the non-histone protein high-mobility group box 1 protein (HMGB1).

Clearly, studies are needed to elucidate the mechanisms of systemic inflammation associated with ACLF, in particular in cases in which a trigger cannot be clinically identified.

\section{ACLF CAN BE AN EARLY MANIFESTATION OF CIRRHOSIS}

An unexpected finding of the CANONIC study is related to the time of development of ACLF in the natural history of cirrhosis. Indeed, this study shows that ACLF develops in the absence of prior history of decompensation in $20 \%$ of cases or develops within few weeks (less than 3 months) after the first episode of decompensation in $\sim 18 \%$ of cases. ${ }^{1}$ These findings indicate that ACLF is not the final event of a long-lasting history of decompensated cirrhosis. In addition, in the ACLF group, patients with no prior history of decompensated cirrhosis develop a more severe form of ACLF, higher levels of inflammation and higher mortality than patients with prior episodes of decompensation.'

\section{CONCLUSIONS}

ACLF is a new syndrome of cirrhosis because it is distinct from "mere" AD. This finding is based not only on the presence of organ failure(s) and high short-term mortality but also on younger age, alcoholic etiology of cirrhosis, higher prevalence of some precipitants (such as bacterial infections, active alcoholism), and more intense systemic inflammatory response. ACLF is a new syndrome also because severe sepsis or severe alcoholic hepatitis do not account for $100 \%$ of the observed cases; in fact, almost $50 \%$ of the cases are of "unknown" origin. In other words, severe sepsis, severe alcoholic hepatitis and ACLF of "unknown origin" are subcategories of the syndrome.

\section{Conflicts of Interest}

The author has no conflicts to disclose.

\section{REFERENCES}

1. Moreau R, Jalan R, Gines P, Pavesi M, Angeli P, Cordoba J, et al. Acute-on-chronic liver failure is a distinct syndrome that develops in patients with acute decompensation of cirrhosis. Gastroenterology 2013;144:1426-1437.

2. Malik R, Mookerjee RP, Jalan R. Infection and inflammation in liver failure: two sides of the same coin. J Hepatol 2009;51:426-429.

3. Wehler M, Kokoska J, Reulbach U, Hahn EG, Strauss R. Short-term prognosis in critically ill patients with cirrhosis assessed by prognostic scoring systems. Hepatology 2001;34:255-261.

4. Das V, Boelle PY, Galbois A, Guidet B, Maury E, Carbonell N, et al. Cirrhotic patients in the medical intensive care unit: early prognosis and long-term survival. Crit Care Med 2010;38:2108-2116.

5. Levesque $E$, Hoti E, Azoulay D, Ichaï $P$, Habouchi $H$, Castaing $D$, et al. Prospective evaluation of the prognostic scores for cirrhotic patients admitted to an intensive care unit. J Hepatol 2012;56:95-102.

6. D'Amico G, Garcia-Tsao G, Pagliaro L. Natural history and prognostic indicators of survival in cirrhosis: a systematic review of 118 studies. J Hepatol 2006;44:217-231.

7. Sarin SK, Kumar A, Almeida JA, Chawla YK, Fan ST, Garg H, et al. Acute-on-chronic liver failure: consensus recommendations of the Asian Pacific Association for the Study of the Liver (APASL). Hepatol Int 2009;3:269-282.

8. Jalan R, Gines P, Olson JC, Mookerjee RP, Moreau R, Garcia-Tsao G, et al. Acute-on-chronic liver failure. J Hepatol 2012 57:1336-1348.

9. Wlodzimirow KA, Eslami S, Abu-Hanna A, Nieuwoudt M, Chamuleau RA. A systematic review on prognostic indicators of acute on chronic liver failure and their predictive value for mortality. Liver Int 2013;33:40-52.

10. Gustot T, Fernandez J, Garcia E, Morando F, Caraceni P, Alessandria $C$, et al. Clinical Course of acute-on-chronic liver failure syndrome and effects on prognosis. Hepatology 2015;62:243-252.

11. Arroyo V, Moreau R, Jalan R, Ginès P, EASL-CLIF Consortium CANONIC Study. Acute-on-chronic liver failure: A new syndrome that will re-classify cirrhosis. J Hepatol 2015;62(1 Suppl):S131-S143.

12. Vincent JL, Moreno R, Takala J, Willatts S, De Mendonça A, Bruining $\mathrm{H}$, et al. The SOFA (Sepsis-related Organ Failure Assessment) score to describe organ dysfunction/failure. On behalf of the Working Group on Sepsis-Related Problems of the European Society of Intensive Care Medicine. Intensive Care Med 1996;22:707-710.

13. Jalan R, Saliba F, Pavesi $M$, Amoros $A$, Moreau $R$, Ginès $P$, et al. Development and validation of a prognostic score to predict mortality in patients with acute-on-chronic liver failure. J Hepatol 2014;6:1038-1047.

14. Li H, Xia Q, Zeng B, Li ST, Liu H, Li Q, et al. Submassive hepatic necrosis distinguishes $\mathrm{HBV}$-associated acute on chronic liver failure from cirrhotic patients with acute decompensation. J Hepatol 2015;63:50-59.

15. Bajaj JS, O'Leary JG, Reddy KR, Wong F, Biggins SW, Patton H, et al. Survival in infection-related acute-on-chronic liver failure is defined by extrahepatic organ failures. Hepatology 2014;60:250-256. 
16. Shi $Y$, Yang $Y$, Hu Y, Wu W, Yang Q, Zheng M, et al. Acute-onchronic liver failure precipitated by hepatic injury is distinct from that precipitated by extrahepatic insults. Hepatology 2015;62:232242.

17. Bernsmeier C, Pop OT, Singanayagam A, Triantafyllou E, Patel VC, Weston CJ, et al. Patients with acute-on-chronic liver failure have increased numbers of regulatory immune cells expressing the receptor tyrosine kinase MERTK. Gastroenterology 2015;148:603-615, e14.

18. Medzhitov R, Schneider DS, Soares MP. Disease tolerance as a defense strategy. Science 2012;335:936-941.

19. Gustot T, Durand F, Lebrec D, Vincent JL, Moreau R. Severe sepsis in cirrhosis. Hepatology 2009;50:2022-2033.

20. Bernardi M, Moreau R, Angeli P, Schnabl B, Arroyo V. Mechanisms of decompensation and organ failure in cirrhosis: From peripheral arterial vasodilation to systemic inflammation hypothesis. J Hepatol 2015;63:1272-1284.

21. Jalan R, Fernandez J, Wiest R, Schnabl B, Moreau R, Angeli P, et al. Bacterial infections in cirrhosis: a position statement based on the EASL Special Conference 2013. J Hepatol 2014;60:1310-1324.

22. Dominguez M, Miquel R, Colmenero J, Moreno M, García-Pagán $J C$, Bosch J, et al. Hepatic expression of CXC chemokines predicts portal hypertension and survival in patients with alcoholic hepatitis. Gastroenterology 2009;136:1639-1650.

23. Lucey MR, Mathurin P, Morgan TR. Alcoholic hepatitis. N Engl J Med 2009;360:2758-2769.

24. Chovatiya R, Medzhitov R. Stress, inflammation, and defense of homeostasis. Mol Cell 2014;54:281-288.

25. Kono $\mathrm{H}$, Rock KL. How dying cells alert the immune system to danger. Nat Rev Immunol 2008;8:279-289. 